\title{
Temporal trends of intraventricular hemorrhage of prematurity in Nova Scotia from 1993 to 2012
}

\author{
Julia A. E. Radic, MD, MPH, ${ }^{1}$ Michael Vincer, MD, ${ }^{2}$ and P. Daniel McNeely, MD1 \\ 1Department of Surgery, Division of Neurosurgery, and ${ }^{2}$ Department of Pediatrics, Division of Neonatal Pediatrics, \\ Dalhousie University, Halifax, Nova Scotia, Canada
}

\begin{abstract}
OBJECT Intraventicular hemorrhage (IVH) and posthemorrhagic hydrocephalus ( $\mathrm{PHH}$ ) are common in premature newborns. The epidemiology of these conditions has been described, but selection bias remains a significant concern in many studies. The goal of this study was to review temporal trends in the incidence of IVH, $\mathrm{PHH}$, and shunt surgery in a population-based cohort of very preterm infants with no selection bias.
\end{abstract}

METHODS All very preterm infants (gestational age $\geq 20$ and $\leq 30$ weeks) born from 1993 onward to residents of Nova Scotia were evaluated by the IWK Health Centre's Perinatal Follow-Up Program, and were entered in a database. Infants born to residents of Nova Scotia from January 1, 1993, to December 31, 2012, were included in this study. The incidences of $\mathrm{IVH}, \mathrm{PHH}$, and shunt surgery were calculated, basic demographic information was described, and chi-square test for trends over time was determined.

RESULTS Of 1334 successfully resuscitated very preterm infants who survived to their initial screening ultrasound, $407(31 \%)$ had an IVH, and 149 (11\%) had an IVH Grade 3 or 4 . No patients with IVH Grade 1 or 2 developed PHH. The percentage of very preterm infants with IVH Grade 3 or 4 has significantly increased over time $(p=0.013)$, as have the incidence of $\mathrm{PHH}$ and shunt surgery $(p=0.001$ and $p=0.011$, respectively) in infants with Grade 3 or $4 \mathrm{IVH}$. The proportion of patients with $\mathrm{PHH}$ receiving a shunt has not changed over time $(p=0.813)$.

CONCLUSIONS The increasing incidence of high-grade IVH-and $\mathrm{PHH}$ and shunt surgery in infants with high-grade $\mathrm{IVH}-$ over time is worrisome. This study identifies a number of associated factors, but further research to identify preventable and treatable causal factors is warranted.

http://thejns.org/doi/abs/10.3171/2014.11.PEDS14363

KEY WORDS intraventricular hemorrhage; hydrocephalus; shunt; prematurity; trend; vascular disorders

$\mathrm{V}$ ERY preterm infants (infants born at less than 31 weeks' gestational age) are at the highest risk of developing intraventricular hemorrhage (IVH) of prematurity. Approximately $15 \%-20 \%$ of very preterm infants develop IVH, ${ }^{5}$ which is typically diagnosed using bedside cranial ultrasound. The severity of the hemorrhage is usually described using the Papile grading system ${ }^{10}$ as Grades $1-4.6,20$

Current literature suggests that approximately $33 \%$ of infants with IVH of prematurity will develop posthemorrhagic ventricular dilation, defined radiographically as having ventricles that are larger than the 97th percentile for the estimated gestational age., ${ }^{4,7} \mathrm{~A}$ recent review of the literature found that $10 \%$ of patients who develop any grade of IVH and $20 \%$ of patients who develop severe IVH develop posthemorrhagic hydrocephalus (PHH), defined as ventricular dilation and symptoms and signs of raised intracranial pressure following a diagnosis of IVH..${ }^{13}$ Signs of raised intracranial pressure include a rapidly enlarging head circumference, splaying of the cranial sutures, and a full fontanel. Many of these infants ultimately require a permanent shunt for CSF diversion, with lifelong implications. ${ }^{13}$

A significant limitation of the literature on this subject is that the major studies on this topic ${ }^{13}$ are not population based, since they are either retrospective reviews at specific institutions or relied upon analyzing data from the National Institutes of Health's National Institute of Child

ABBREVIATIONS CPR = cardiopulmonary resuscitation; IVH = intraventricular hemorrhage; $\mathrm{NICU}=$ neonatal intensive care unit; $\mathrm{PHH}=$ posthemorrhagic hydrocephalus; $\mathrm{VAD}=$ ventricular assist device.

SUBMITTED July 13, 2014. ACCEPTED November 24, 2014.

INCLUDE WHEN CITING Published online March 13, 2015; DOI: 10.3171/2014.11.PEDS14363.

DISCLOSURE The authors report no conflict of interest concerning the materials or methods used in this study or the findings specified in this paper. 
Health and Human Development Neonatal Research Network to provide a multicenter snapshot based on the experience of select academic centers. Therefore, there could be significant selection bias impacting the validity of these epidemiology reports.

The objective of this study was to accurately describe the trends in incidence of IVH and subsequent PHH/shunt surgery over time, by studying a population-based cohort of very preterm (gestational age $\geq 20$ and $\leq 30$ weeks) infants born to residents of Nova Scotia from January 1, 1993, to December 31, 2012, using a prospective database with minimal selection bias.

\section{Methods}

All very preterm ( $\leq 30$ completed weeks' gestational age) infants born to residents of Nova Scotia from January 1,1993, onward were included in a comprehensive database. Inclusion criteria for this study included the following: born to a resident of Nova Scotia between January 1,1993 , and December 31, 2012, gestational age of $\leq$ 30 weeks but $\geq 20$ weeks, and successfully resuscitated at birth. Exclusion criteria included: missing information about IVH status (which only occurred when an infant died before the initial 3-day postnatal screening ultrasound and also no autopsy was performed).

Dr. Michael Vincer, who has been in charge of this database and the perinatal follow-up program for the duration of the study period, actively tracks all preterm births in Nova Scotia. The majority of these patients are managed at our center. In addition, Dr. Vincer regularly visits the only 2 other regional neonatal units that manage preterm infants of Nova Scotian mothers (in Moncton, New Brunswick, and in Sydney, Nova Scotia) to collect data on the preterm infants treated at these sites. Preterm infants managed outside of Moncton, Sydney, or Halifax (for example, one born to a Nova Scotian mother while she was traveling elsewhere on vacation) would also be routinely referred to the perinatal follow-up program at the IWK Health Centre in Halifax by their pediatrician or family doctor once the infant and mother returned to Nova Scotia.

The imaging screening protocol has remained largely unchanged since 1993 in Nova Scotia. Very preterm infants universally undergo cranial ultrasonography screening examinations within 3 days of birth, at 2 weeks, at 6 weeks, and again at term. The method of ultrasound screening did change slightly starting in 2010 to include examining the brain through multiple approaches in addition to through the anterior fontanel. Furthermore, since 2010, some infants have been examined with MRI brain instead of an ultrasound examination at term.

The resuscitation protocol has undergone few changes over time. A formalized neonatal resuscitation program was first initiated approximately 20 years ago. In 1999 , a practice of wrapping babies in polyethylene occlusive wrap $^{19}$ was started, and this became standard practice in 2000 . In the past 10 years, there has been a reduction in oxygen use in the delivery room. Also, 15 years ago, respiratory therapists were added to the resuscitation team.

$\mathrm{PHH}$ was defined as the presence of ventricles larger than the 97th percentile for estimated gestational age in in- fants who also had symptoms and signs of raised intracranial pressure (enlarging head circumference, splaying of the cranial sutures, and a full fontanel) following a diagnosis of IVH. Infants with PHH received a permanent shunt if they reached a weight of $2 \mathrm{~kg}$ and continued to have a diagnosis of PHH. Ventricular access devices (VADs), or watchful waiting, were used to bridge the gap in between diagnosis of $\mathrm{PHH}$ and weight of $2 \mathrm{~kg}$, at the discretion of the surgeon. Infants whose ventricle dimensions and clinical status stabilized or improved by the time they reached $2 \mathrm{~kg}$ in weight did not receive a permanent shunt.

The numbers of infants who were successfully resuscitated at birth, who were diagnosed with IVH, who developed $\mathrm{PHH}$, and who were treated with a shunt for CSF diversion by their final follow-up between 2 and 3 years of age, were extracted from the database (Fig. 1). The number of infants who were treated with a VAD was also determined, as was the percentage of infants who were lost to final follow-up after discharge from the neonatal intensive care unit (NICU).

The incidence of IVH (by Papile grade ${ }^{4}$ ) and of $\mathrm{PHH}$, VAD use, and shunt surgery in all very preterm infants born to residents of Nova Scotia from January 1, 1993, to December 31, 2012, was calculated for this cohort. A chi-square test for trend was used to assess for changing incidence of IVH, PHH, VAD use, and shunt surgery over time, with time broken down into 5-year epochs.

Odds ratios for IVH were calculated and assessed for significance using univariate logistic regression for several perinatal, infant, and maternal variables, including use of antenatal steroids, birth weight, cardiopulmonary resuscitation, inotrope usage, gestational age, infant respiratory distress syndrome, cesarean section, maternal age, maternal weight, maternal height, infant sex, surfactant use, use of high-flow oscillatory ventilation, ventilation use, infant death in the first 7 days, neonatal death, gestational hypertension, maternal fever during labor, suspected intrauterine growth restriction on ultrasonography, birth asphyxia, necrotizing enterocolitis, overall chance of resuscitation at birth, intrapartum magnesium sulfate use, twin pregnancy, nitric oxide use, use of a paralyzing agent, phototherapy, and cystic periventricular leukomalacia. Univariate linear regression was used to assess for significant associations between each of these variables and time (in 5-year epochs).

Multivariate logistic regression was used to assess for a significant association between IVH and time, as well as Grade 3 or 4 IVH and time, controlling first for death in the first 7 days, and second for perinatal, infant, and maternal variables that were significantly associated with both IVH and time and that were clinically determined to occur primarily before the diagnosis of IVH.

Multivariate logistic regression was also used to determine whether hydrocephalus and CSF shunting following a Grade 3 or 4 IVH were significantly associated with time, when controlling for mortality.

All data analysis was carried out using STATA 11.2 (StataCorp LP). Results were considered statistically significant if they had a $p$ value $<0.05$. Ethics approval for this research was obtained from the IWK Health Centre Research Ethics Board. 


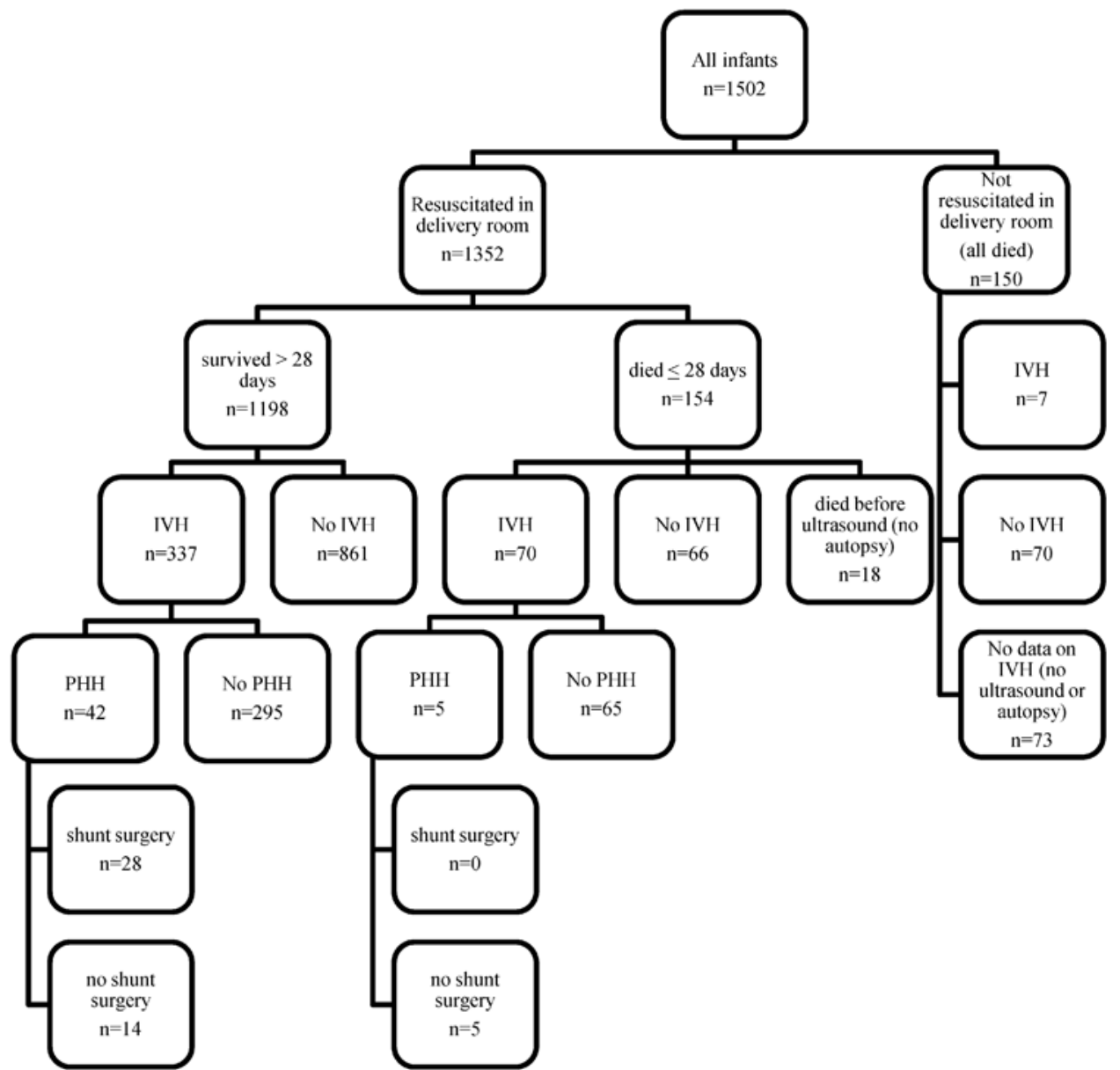

FIG. 1. Distribution of IVH, PHH, and shunt surgery in very preterm infants born to residents of Nova Scotia between January 1 , 1993, and December 31, 2012.

\section{Results}

\section{Intraventricular Hemorrhage}

A total of 1502 very preterm infants $(\geq 20$ and $\leq 30$ weeks gestational age) were born to residents of Nova Scotia from January 1, 1993, to December 31,2012, and of these, $1352(90 \%)$ infants were successfully resuscitated. Of these, 18 died within the first 13 hours of life before receiving a screening ultrasound and without receiving an autopsy, leaving a total of 1334 infants. Of these, 407 $(31 \%)$ infants received a diagnosis of IVH and $170(13 \%)$, $88(7 \%), 62(5 \%)$, and $87(7 \%)$ a diagnosis of IVH Grade $1,2,3$, and 4 , respectively. Thirty infants $(2 \%)$ were lost to follow-up after discharge from the NICU (Fig. 1).

The percentage of successfully resuscitated very preterm infants with IVH of any grade has not changed significantly over time ( $\mathrm{p}=0.051)$ from $27 \%$ (102 of 374 infants) during 1993-1997 to 35\% (118 of 342 during 2008-2012. However, the percentage with IVH Grade 3 or 4 in particular has significantly increased over time ( $\mathrm{p}$ $=0.013$ ), from $8 \%$ (31 of 374 infants) during 1993-1997 to $15 \%$ (50 of 342) during 2008-2012 (Fig. 2).

A list of various infant, maternal, and management variables, their associations with IVH, and their change in incidence over time can be found in Table 1. Note that, in particular, the percentage of very preterm infants who were successfully resuscitated has not changed significantly over time ( $p=0.279)$, and the gestational age of successfully resuscitated very preterm infants was not significantly different between the first 5 years and the last 5 years of this study $(\mathrm{p}=0.404)$. On the other hand, both 7-day and 28-day mortality in successfully resuscitated very preterm infants have significantly decreased over

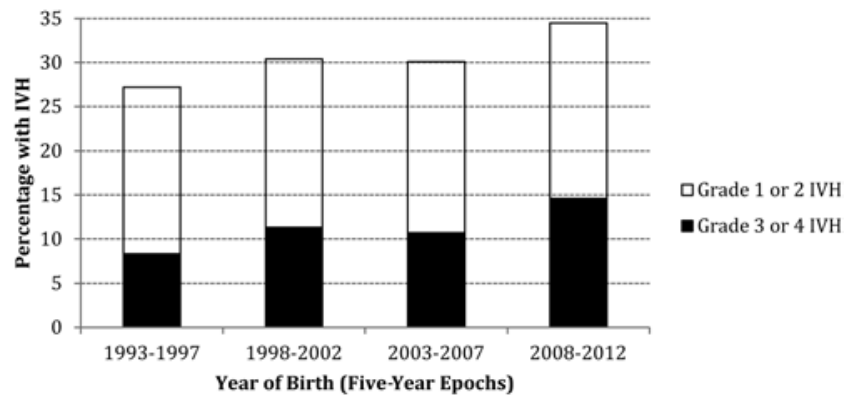

FIG. 2. Percentage of successfully resuscitated very preterm infants with IVH by date of birth in 5-year intervals. Mild IVH: Grades 1 or 2 , severe IVH: Grades 3 or 4 . Chi-square test for trend for any grade of IVH, $p=0.051$; for Grades 3 or 4 IVH, $p=0.013$. 
TABLE 1. Perinatal, infant, and maternal variables and their association with IVH and an analysis of whether the risk of these variables has changed over time*

\begin{tabular}{|c|c|c|c|c|c|c|c|}
\hline \multirow[b]{2}{*}{ Variable } & \multirow{2}{*}{$\begin{array}{l}\text { OR† } \\
\text { for } \\
\text { IVH }\end{array}$} & \multirow{2}{*}{$\begin{array}{c}\text { p Value }{ }^{\text {of }} \\
\text { Assoc btwn } \\
\text { IVH \& Variable }\end{array}$} & \multicolumn{4}{|c|}{ Risk or or Mean Value of Variable } & \multirow{2}{*}{$\begin{array}{c}\text { p Value } \text { of } \\
\text { Assoc btwn } \\
\text { Variable \& Time }\end{array}$} \\
\hline & & & $\begin{array}{l}1993-1997 \\
(n=374)\end{array}$ & $\begin{array}{c}1998-2002 \\
(n=308)\end{array}$ & $\begin{array}{c}2003-2007 \\
(n=310)\end{array}$ & $\begin{array}{c}2008-2012 \\
(n=342)\end{array}$ & \\
\hline Antenatal corticosteroids given & 0.60 & 0.002 & 0.81 & 0.87 & 0.90 & 0.89 & 0.001 \\
\hline Birth weight (g) & 1.00 & $<0.001$ & $1056 \pm 308.9$ & $1116 \pm 363.6$ & $1117 \pm 338.6$ & $1114 \pm 357.0$ & 0.027 \\
\hline $\begin{array}{l}\text { Any cardiopulmonary resuscita- } \\
\text { tion }\end{array}$ & 2.50 & $<0.001$ & 0.04 & 0.07 & 0.05 & 0.13 & $<0.001$ \\
\hline Dobutamine use & 2.65 & $<0.001$ & 0.02 & 0.02 & 0.04 & 0.10 & $<0.001$ \\
\hline Dopamine use & 2.88 & $<0.001$ & 0.14 & 0.16 & 0.19 & 0.25 & $<0.001$ \\
\hline Gestational age (wks) & 0.81 & $<0.001$ & $27.7 \pm 2.1$ & $27.7 \pm 2.1$ & $27.7 \pm 1.9$ & $27.6 \pm 2.2$ & 0.479 \\
\hline $\begin{array}{l}\text { Infant respiratory distress syn- } \\
\text { drome }\end{array}$ & 1.38 & 0.097 & 0.83 & 0.87 & 0.87 & 0.95 & $<0.001$ \\
\hline Cesarean section & 0.40 & $<0.001$ & 0.56 & 0.50 & 0.58 & 0.57 & 0.340 \\
\hline Maternal age (yrs) & 0.97 & 0.011 & $28.1 \pm 5.7$ & $28.6 \pm 6.3$ & $28.8 \pm 5.5$ & $28.7 \pm 5.4$ & 0.126 \\
\hline Maternal weight (kg) & 1.00 & 0.401 & $65.4 \pm 13.4$ & $68.0 \pm 17.4$ & $70.7 \pm 17.9$ & $69.5 \pm 18.3$ & 0.001 \\
\hline Maternal height (m) & 0.98 & 0.985 & $1.63 \pm 0.07$ & $1.63 \pm 0.07$ & $1.65 \pm 0.07$ & $1.63 \pm 0.08$ & 0.042 \\
\hline Male sex & 1.31 & 0.024 & 0.49 & 0.53 & 0.53 & 0.54 & 0.189 \\
\hline Surfactant use & 1.58 & 0.001 & 0.56 & 0.71 & 0.76 & 0.77 & $<0.001$ \\
\hline High-flow oscillatory ventilation & 2.90 & $<0.001$ & 0.02 & 0.08 & 0.16 & 0.13 & $<0.001$ \\
\hline Ventilation & 1.48 & 0.006 & 0.68 & 0.83 & 0.83 & 0.70 & 0.544 \\
\hline $\begin{array}{l}\text { Infant death in the first } 7 \text { days } \\
\text { of life }\end{array}$ & 2.15 & $<0.001$ & 0.13 & 0.09 & 0.05 & 0.03 & $<0.001$ \\
\hline Neonatal death & 2.71 & $<0.001$ & 0.16 & 0.11 & 0.08 & 0.06 & $<0.001$ \\
\hline Gestational hypertension & 0.59 & 0.002 & 0.17 & 0.17 & 0.18 & 0.18 & 0.788 \\
\hline $\begin{array}{l}\text { Maternal fever during labor (temp } \\
\quad>38^{\circ} \mathrm{C} \text { ) }\end{array}$ & 2.00 & 0.009 & 0.09 & 0.06 & 0.02 & 0.01 & $<0.001$ \\
\hline $\begin{array}{l}\text { Suspected intrauterine growth } \\
\text { restriction on ultrasound }\end{array}$ & 0.48 & 0.046 & 0.06 & 0.03 & 0.03 & 0.03 & 0.038 \\
\hline Birth asphyxia & 1.56 & 0.001 & 0.62 & 0.62 & 0.79 & 0.85 & $<0.001$ \\
\hline Necrotizing enterocolitis & 2.58 & 0.002 & 0.02 & 0.02 & 0.02 & 0.07 & 0.001 \\
\hline $\begin{array}{l}\text { Resuscitation (in all very preterm } \\
\text { infants) }\end{array}$ & 4.39 & $<0.001$ & 0.88 & 0.90 & 0.92 & 0.90 & 0.279 \\
\hline $\begin{array}{l}\text { Intrapartum magnesium sulfate } \\
\text { use }\end{array}$ & 0.51 & $<0.001$ & 0.25 & 0.22 & 0.09 & 0.30 & 0.877 \\
\hline Twin pregnancy & 0.83 & 0.160 & 0.24 & 0.25 & 0.32 & 0.30 & 0.018 \\
\hline Nitric oxide use in the baby & 2.62 & $<0.001$ & 0.01 & 0.06 & 0.07 & 0.11 & $<0.001$ \\
\hline Paralyzing agent & 2.58 & $<0.001$ & 0.06 & 0.06 & 0.06 & 0.29 & $<0.001$ \\
\hline Phototherapy & 0.88 & 0.530 & 0.10 & 0.10 & 0.06 & 0.14 & 0.173 \\
\hline Cystic & 4.68 & $<0.001$ & 0.08 & 0.06 & 0.05 & 0.08 & 0.763 \\
\hline
\end{tabular}

Assoc = association; temp = temperature; $\mathrm{VL}=$ periventricular leukomalacia.

* Bold type indicates statistical significance.

$\dagger$ Risk values are given for discrete variables and mean values $( \pm S D)$ are given for continuous variables.

$\ddagger$ Calculated using univariate logistic regression.

$\S$ Calculated using univariate linear regression.

time ( $\mathrm{p}<0.001, \mathrm{p}<0.001)$. Multivariable logistic regression analysis looking at the association between IVH and time, controlling for death in the first 7 days after birth, resulted in a significant association between both IVH of any grade and time (OR 1.14, $\mathrm{p}=0.011)$ and Grade 3 or $4 \mathrm{IVH}$ and time (OR 1.36, $\mathrm{p}<0.001)$. A number of other potential protective factors and risk factors for IVH have also changed significantly over time (Table 1).
From Table 1, the variables that showed a statistically significant association with both IVH and time and that were clinically determined to potentially occur before IVH diagnosis were antenatal corticosteroid use, birth weight, cardiopulmonary resuscitation (CPR), dobutamine use, dopamine use, surfactant use, high-flow oscillatory ventilation use, maternal fever during labor, suspected intrauterine growth restriction on ultrasonography, birth 


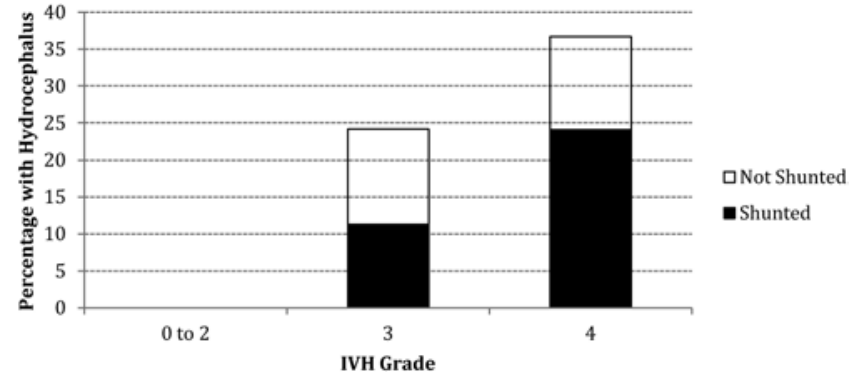

FIG. 3. Percentage of successfully resuscitated very preterm infants with $\mathrm{PHH}$ by grade of IVH and by whether they were shunted, born between January 1, 1993, and December 31, 2012.

asphyxia, nitric oxide use in the baby, paralyzing agent use, and death in the 1st week after birth. On multivariate logistic regression analysis including these variables in the model, there continued to be no significant association between IVH of any grade and time. However, in addition to this, Grade 3 or 4 IVH was no longer significantly associated with time $(\mathrm{OR} 1.06, \mathrm{p}=0.319)$. The variables that remained independently associated with Grade 3 or 4 IVH included antenatal steroid use (OR 0.50, $\mathrm{p}=0.003)$, infant CPR (OR 2.38, $\mathrm{p}=0.002)$, dopamine use (OR 2.23, $\mathrm{p}<$ $0.001)$, maternal fever during labor (OR 4.97, $\mathrm{p}<0.001)$, and death in the first 7 days (OR 2.90, $\mathrm{p}<0.001)$.

\section{Hydrocephalus and Shunt Surgery}

Of successfully resuscitated infants born between January 1, 1993, and December 31, 2012, $0(0 \%)$ of 170 infants with Grade 1 IVH, 0 ( $0 \%$ ) of 88 infants with Grade 2 IVH, $15(24 \%)$ of 62 infants with Grade 3 IVH, and $32(37 \%)$ of 87 infants with Grade 4 IVH, compared with $0(0 \%)$ of 927 infants without IVH, were diagnosed with hydrocephalus (Fig. 3). The percentage of very preterm infants with Grade 3 or 4 IVH who were diagnosed with hydrocephalus has increased over time $(\mathrm{p}=0.001)$, from $16 \%(5$ of 31 infants) during 1993-1997 to 48\% (24 of 50) during 2008-2012 (Fig. 4). On multivariate logistic regression analysis, controlling for mortality, hydrocephalus remains significantly associated with time (OR 1.64, $\mathrm{p}=0.005)$.

Also, 0 (0\%) of 170 infants with Grade 1 IVH, $0(0 \%)$ of 88 infants with Grade 2 IVH , 7 (11\%) of 62 with Grade $3 \mathrm{IVH}$, and 21 (24\%) of 87 with Grade 4 IVH, compared with $0(\%)$ of 927 without IVH, were treated with a shunt for hydrocephalus (Fig. 3). The percentage of very preterm infants with Grade 3 or 4 IVH who were treated with a shunt has also increased over time $(\mathrm{p}=0.011)$, from $10 \%(3$ of 31) during 1993-1997 to 30\% (15 of 50) during 20082012 (Fig. 4). On multivariate logistic regression analysis, controlling for mortality, shunting remains significantly associated with time (OR 1.51, $\mathrm{p}=0.049$ ). The percentage of very preterm infants with hydrocephalus who were treated with a shunt $(28 / 47,60 \%)$ has not changed over time $(\mathrm{p}=0.813)$ (Fig. 5).

Furthermore, $2(13 \%)$ of 15 and 8 (21\%) of 32 infants with hydrocephalus following Grade 3 and Grade 4 IVH, respectively, were treated with a VAD over the study period; 9 of 10 infants with a VAD went on to need a perma-

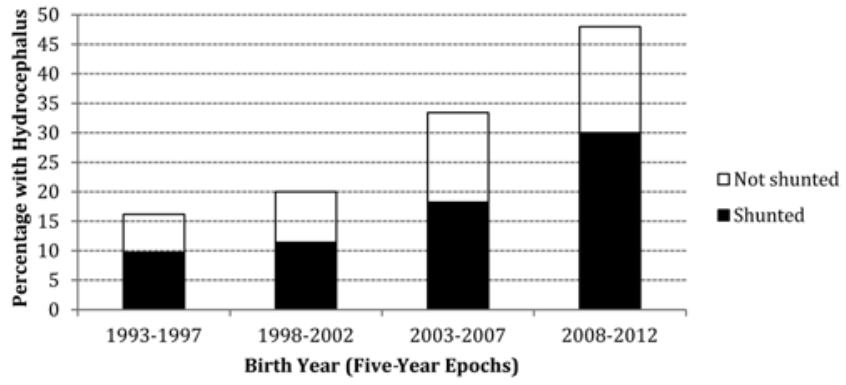

FIG. 4. Percentage of successfully resuscitated very preterm infants with Grade 3 or 4 IVH born between January 1, 1993, and December 31, 2012, who develop PHH by whether they were shunted. Chi-square test for $\mathrm{PHH}$ is $p=0.001$ and for shunt surgery is $p=0.011$.

nent shunt, and 1 infant with Grade 3 IVH improved and did not require a shunt. Of the other 37 infants with PHH who were not treated with a VAD (13 of 15 with Grade 3 IVH and 24 of 32 with Grade 4 IVH), 18 improved and did not require a shunt, and 19 went on to require a permanent shunt. In $8(80 \%)$ of the 10 cases in which VADs were used, the patients were treated between 2008 and 2012; in the other 2 cases, the patients were treated between 1998 and 2002. The percentage of very preterm infants with hydrocephalus who were treated with a VAD (10 [21\%] of 47 ) has not significantly changed over time $(p=0.123)$.

\section{Discussion}

This population-based study confirms that IVH is a common diagnosis in very preterm infants, with $31 \%$ of successfully resuscitated very preterm infants developing IVH of any grade, and $11 \%$ developing IVH Grade 3 or 4. In addition, in Nova Scotia, the incidence of IVH Grades 3 or 4 appears to be significantly increasing in very preterm infants over time. This observation is in contrast with other studies that have reported a stable ${ }^{2,8}$ or decreasing ${ }^{12,13,16}$ incidence of IVH of prematurity, though some centers report an increasing incidence. ${ }^{11}$ None of these studies are population based and are therefore subject to referral bias. Furthermore, some represent more historical time periods, ${ }^{2,12,16}$ and are therefore not directly comparable to this study.

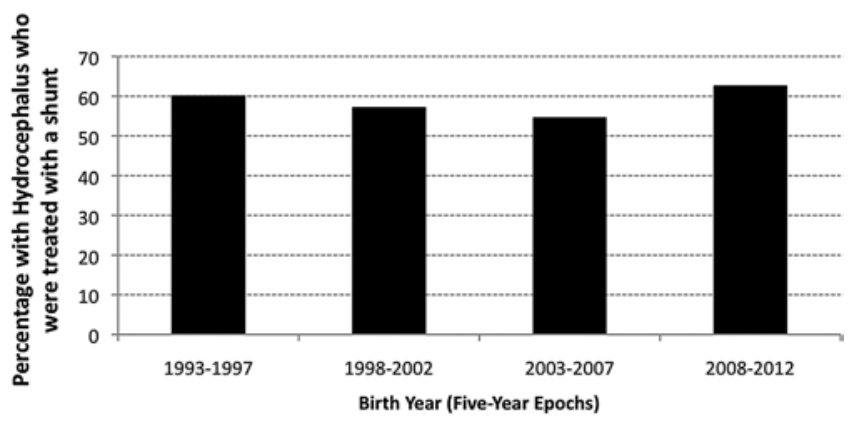

FIG. 5. Percentage of successfully resuscitated very preterm infants with PHH born between January 1, 1993, and December 31, 2012, who were treated with a shunt. Chi-square test for trend is nonsignificant ( $p$ $=0.813$ ). 
The reasons for the increasing incidence of IVH of prematurity in the Nova Scotia population are uncertain. Importantly, the increasing incidence is not explained by changes in diagnostic criteria (which have not changed since 1993 and continue to be based on the Papile grading system). Additionally, the imaging screening protocol has remained unchanged since 1993 in Nova Scotia. Furthermore, although the method of screening changed slightly starting in 2010 to include examining the brain via ultrasonography through multiple approaches in addition to through the anterior fontanel, as well as by occasionally replacing the ultrasonographic examination performed at term with MRI since 2010, this is unlikely to have significantly impacted incidence proportions of Grade 3 and 4 IVHs, and so does not explain the increasing incidence over time.

Other factors were considered as well. Gestational age and proportion of very preterm infants who were successfully resuscitated at birth do not appear to be changing over time and so cannot explain the increasing incidence of Grade 3 or $4 \mathrm{IVH}$ in this cohort. On the other hand, neonatal mortality appears to have decreased over time. It was considered that perhaps more infants were surviving to be diagnosed with IVH than before; however, the association between Grade 3 or 4 IVH and time remains significant when controlling for early mortality in the first 7 days after birth. Furthermore, IVH of any grade becomes significantly associated with time as well, which implies that the association between IVH and time is not solely due to improved survival.

Several variables that are risk factors for IVH ${ }^{14,15,18}$ are increasing in incidence over time in Nova Scotia, which may help explain the increasing IVH incidence. These include infant CPR and inotrope use. Other risk factors are decreasing, such as maternal fever during labor, and other protective factors are increasing in incidence over time, such as antenatal steroid use. From the multivariate logistic regression analysis, it seems that the increasing incidence in IVH could be at least partially explained by the increasing use of CPR and ionotropes and by improved survival in the 1st week after birth. The increase over time in these risk factors could be overriding the increase over time in protective antenatal steroid use and decrease over time in maternal fever during labor, leading to increasing incidence of IVH of prematurity. Further research into the cause of the increasing incidence of IVH is essential, as IVH comes with significant risk of morbidity and mortality. ${ }^{3}$

Interestingly, this study demonstrated that the lower grades of IVH (Grades 1 and 2) are associated with a very low risk (no observed cases in this cohort) of developing $\mathrm{PHH}$ and requiring shunt surgery, in contrast to previous reports, which showed risks as high as a $5 \%$ and $20 \%$ for developing PHH for IVH Grade 1 and Grade 2, respectively. ${ }^{9,17}$ This could be due to referral bias in other studies, as they are not population based. The centers reporting IVH and PHH risks in previously published studies may have been referred only the sickest IVH patients, which may have suggested a disproportionately high risk of hydrocephalus in Grade 2 IVH infants. Our center is referred all infants with IVH, and hence referral bias is unlikely in the present study.
The incidence of both PHH and shunt placement in the group of very preterm infants with Grade 3 or 4 IVH has been increasing over time as well. There has been no significant change over time in the proportion of infants with $\mathrm{PHH}$ who have received a shunt, so the increase in shunt placement over time appears to be due to the increase in PHH risk over time and not due to change in indications for shunt placement over time in infants with PHH. This experience is in contrast to that of other centers that have reported a decreased ${ }^{1}$ or stable ${ }^{8}$ risk of $\mathrm{PHH}$ in infants with severe IVH. Note must be made here that the definition of PHH used by this database has not changed since 1993, so the increasing incidence of hydrocephalus in severe IVH cannot be explained by any such change. Given increasing survival over time to the estimated delivery date, perhaps more infants with IVH are surviving to be diagnosed with clinical hydrocephalus. However, the association between $\mathrm{PHH}$ and time and between shunting and time remains significant even when controlling for improved survival, suggesting that other factors are likely at play. Further research is needed to determine what these factors might be.

This study is limited by the censoring of infants with a gestational age of 31 weeks or more, and so the results of this analysis are only applicable to infants born at less than 31 weeks' gestational age. However, the risk of IVH in infants of 31 weeks' gestational age or older is much lower than in infants born at an earlier gestational age, ${ }^{13}$ and so these study results are likely reflective of the majority of IVH cases in infants born to residents of Nova Scotia during the study period.

The strengths of this study include the extensive efforts made to ensure that every very preterm infant born to a resident of Nova Scotia during the study period was included in this database for the duration of their NICU admission, including infants born at all hospitals that provided NICU care to Nova Scotia residents. All of these infants/families were then offered the opportunity to remain included in the Perinatal Follow-Up Program and database after discharge from the hospital until final follow-up at between 2 and 3 years of age, and the loss-to-follow-up risk was only $2 \%$. Consequently, there is a very low risk of selection bias, which increases the validity of this population-based study and makes the results generalizable to other populations with similar demographics and NICU management protocols.

\section{Conclusions}

In conclusion, IVH, PHH, and shunt treatment are common in very preterm infants, and their incidence has been increasing over time. The reasons for this are unclear, although it may be partially due to improving survival and increasing incidence of various risk factors for IVH. The trend is worrisome, and further study is needed to identify preventable and treatable causal factors.

\section{References}

1. Alan N, Manjila S, Minich N, Bass N, Cohen AR, Walsh M, et al: Reduced ventricular shunt rate in very preterm infants with severe intraventricular hemorrhage: an institutional experience. J Neurosurg Pediatr 10:357-364, 2012 
2. Batton DG, DeWitte DB, Boal DK, Nardis EE, Maisels MJ: Incidence and severity of intraventricular hemorrhage: 19811984. Am J Perinatol 3:353-356, 1986

3. Brouwer AJ, Groenendaal F, Benders MJ, de Vries LS: Early and late complications of germinal matrix-intraventricular haemorrhage in the preterm infant: what is new? Neonatology 106:296-303, 2014

4. de Vries LS, Liem KD, van Dijk K, Smit BJ, Sie L, Rademaker KJ, et al: Early versus late treatment of posthaemorrhagic ventricular dilatation: results of a retrospective study from five neonatal intensive care units in The Netherlands. Acta Paediatr 91:212-217, 2002

5. du Plessis AJ: The role of systemic hemodynamic disturbances in prematurity-related brain injury. J Child Neurol 24:1127-1140, 2009

6. El-Dib M, Massaro AN, Bulas D, Aly H: Neuroimaging and neurodevelopmental outcome of premature infants. Am J Perinatol 27:803-818, 2010

7. Levene MI: Measurement of the growth of the lateral ventricles in preterm infants with real-time ultrasound. Arch Dis Child 56:900-904, 1981

8. Limbrick DD Jr, Mathur A, Johnston JM, Munro R, Sagar J, Inder T, et al: Neurosurgical treatment of progressive posthemorrhagic ventricular dilation in preterm infants: a 10-year single-institution study. J Neurosurg Pediatr 6:224-230, 2010

9. Luciano M, Pattisapu JV, Wickremesekera A: Infantile posthemorhhagic hydrocephalus, in Winn HR (ed): Youmans Neurological Surgery, ed 5. Philadelphia: Saunders, 2004, Vol 4, pp 3405-3417

10. Papile LA, Burstein J, Burstein R, Koffler H: Incidence and evolution of subependymal and intraventricular hemorrhage: a study of infants with birth weights less than 1,500 gm. J Pediatr 92:529-534, 1978

11. Paul DA, Leef KH, Locke RG, Bartoshesky L, Walrath J, Stefano JL: Increasing illness severity in very low birth weight infants over a 9-year period. BMC Pediatr 6:2, 2006

12. Philip AG, Allan WC, Tito AM, Wheeler LR: Intraventricular hemorrhage in preterm infants: declining incidence in the 1980s. Pediatrics 84:797-801, 1989

13. Robinson S: Neonatal posthemorrhagic hydrocephalus from prematurity: pathophysiology and current treatment concepts. J Neurosurg Pediatr 9:242-258, 2012

14. Salafia CM, Minior VK, Rosenkrantz TS, Pezzullo JC, Popek EJ, Cusick W, et al: Maternal, placental, and neonatal associations with early germinal matrix/intraventricular hemorrhage in infants born before 32 weeks' gestation. Am J Perinatol 12:429-436, 1995

15. Sarkar S, Bhagat I, Dechert R, Schumacher RE, Donn SM: Severe intraventricular hemorrhage in preterm infants: comparison of risk factors and short-term neonatal morbidities between grade 3 and grade 4 intraventricular hemorrhage. Am J Perinatol 26:419-424, 2009

16. Strand C, Laptook AR, Dowling S, Campbell N, Lasky RE, Wallin LA, et al: Neonatal intracranial hemorrhage: I. Changing pattern in inborn low-birth-weight infants. Early Hum Dev 23:117-128, 1990

17. Tsitouras V, Sgouros S: Infantile posthemorrhagic hydrocephalus. Childs Nerv Syst 27:1595-1608, 2011

18. Vergani P, Patanè L, Doria P, Borroni C, Cappellini A, Pezzullo JC, et al: Risk factors for neonatal intraventricular haemorrhage in spontaneous prematurity at 32 weeks gestation or less. Placenta 21:402-407, 2000

19. Vohra S, Frent G, Campbell V, Abbott M, Whyte R: Effect of polyethylene occlusive skin wrapping on heat loss in very low birth weight infants at delivery: a randomized trial. J Pediatr 134:547-551, 1999

20. Volpe JJ: Neurology of the Newborn, ed 5. Philadelphia: Saunders Elsevier, 2008

\section{Author Contributions}

Conception and design: all authors. Acquisition of data: Vincer. Analysis and interpretation of data: Radic. Drafting the article: Radic. Critically revising the article: all authors. Reviewed submitted version of manuscript: all authors. Approved the final version of the manuscript on behalf of all authors: Radic. Statistical analysis: Radic. Study supervision: Vincer, McNeely.

\section{Supplemental Information \\ Previous Presentation}

Portions of this work were presented in platform presentation form at the Canadian Pediatric Neurosurgery Research Study Group annual meeting in Whistler, British Columbia, on February 27, 2014, and at the Canadian Neurological Sciences Federation meeting in Banff, Alberta, on June 6, 2014.

\section{Correspondence}

Julia A. E. Radic, Division of Neurosurgery, IWK Health Centre, 5850 University Ave., Halifax, NS B3K 6R8, Canada. email: jradic@dal.ca. 